\title{
Los libros de ajedrez en España (c. 1770-1899): bibliografía histórica
}

\author{
Jordi Brasó i Rius*
}

Artículo recibido:

23 de febrero de 2015

Artículo aceptado:

27 de septiembre de 2016

\section{Resumen}

Se presenta un repertorio bio-bibliográfico y un estudio bibliométrico histórico de las obras de ajedrez en España, desde finales del siglo XVIII hasta finales del siglo XIX. Se ha utilizado una metodología basada en la búsqueda y análisis en los fondos documentales de la época y en las bases de datos de las bibliotecas actuales, principalmente de España. La fase interpretativa, junto con un análisis estadístico de cuatro factores -periodo de publicación de las obras, lugar de edición, originalidad o traducción, y tipología- permite describir cada una de las obras de ajedrez publicadas en España, además de contextualizarlas con el momento social de la época. El estudio bibliométrico refuerza

* Centre d'Alt Rendiment de Sant Cugat del Vallès, Barcelona, Catalunya, España jbraso@xtec.cat_jordibrasorius@gmail.com

INVESTIGACIÓN BIBLIOTECOLÓGICA, Vol. 32, Núm. 75, abril/junio, 2018, México, ISSN: 2448-8321. pp. 47-67 
así el análisis interpretativo sobre la producción ajedrecística española.

Palabras clave: Ajedrez; Análisis Bibliométrico; Bibliografía; Bibliometría Histórica; Bio-Bibliografía; España; Siglo XIX

\section{Chess books in Spain (1770-1899): Historical bibliography Jordi Brasó $i$ Rius}

\section{Abstract}

This paper offers a bio-bibliographic repertory and historical bibliometric study of works on chess in Spain from the late eighteenth century to the late nineteenth century. The methodology employed entails research and analysis of documentary sources of the period and current library databases mainly in Spain. The subsequent interpretative phase and the bibliometric analysis of four factors (period of publication, place of publication, originality vs. translation and typology) serves to describe each of the chess works published in Spain. Moreover, it also allows contextualization of the works within the social milieu. In this way, the bibliometric study reinforces the interpretative analysis of Spanish chess production.

Keywords: Bibliometric Study; Bibliography; BioBibliography; Chess; Historical Bibliometrics; Nineteenth Century Spain

\section{INTRODUCCIÓN}

T a historia de los deportes y los juegos permite analizar diferentes aspecLtos para entender las sociedades de cada época. Es además conveniente tratar de forma específica cada una de estas aficiones. Uno de estos campos de análisis es el ajedrez, practicado desde tiempos remotos pero a la vez poco estudiado, en relación con la mayoría de disciplinas lúdicas y deportivas.

En esta línea, cualquier rama del conocimiento requiere de repertorios y catálogos bibliográficos que permitan conocer la evolución histórica y dar rigor científico a cada una de estas disciplinas (Blanco, 1927; Brasó, 2016; Menéndez Pelayo, 1876; Olivera, 2011; Pastor, 1995; Torrebadella, 2014; 
Torrebadella y Nomdedeu, 2014; Torrebadella y Olivera, 2012). Estas obras se hacen necesarias a partir de la aparición de la imprenta, que significó un incremento de la divulgación de la cultura a partir de los libros, de lo que deriva la necesidad de registrar estas publicaciones. Además, estas compilaciones permiten conocer el "pensamiento religioso, filosófico, etc. de su tiempo” (Punzano, 1981: 150).

Así, haciendo frente al menosprecio que aparece en el siglo XIX por la cultura popular, es destacable un movimiento encabezado por la figura de Menéndez Pelayo (1876), declarando que es necesario recordar el pasado y valorar la tradición como un elemento que permite un efectivo progreso en todos los ámbitos. Menéndez Pelayo (1876) reitera la exageración innovadora y reaccionaria que hay en España, basada en estudios extranjeros sin tener en cuenta los autóctonos, y pretende hacerle frente principalmente mediante los estudios bibliográficos e históricos (Bolado, 2012; Menéndez Pelayo, 1876).

Por lo tanto, se empieza a considerar la bibliografía como esencial y base del conocimiento científico (Menéndez Pelayo, 1876; Gallego, 2005; Punzano, 1981; Simón Díaz, 1977). Menéndez Pelayo detalla además que dichos estudios no se tendrían que limitar solamente a tratar los aspectos externos de las obras, sino que también tendrían que tratar y reflexionar sobre el contenido en sí de los libros. Por lo tanto, la "bibliografía es al mismo tiempo el cuerpo, la historia externa del movimiento intelectual, y una preparación excelente e indispensable para el estudio de la historia interna" (Menéndez Pelayo, 1876: 66). También reflexiona el autor sobre la cantidad de bibliografías y bio-bibliografías producidas en España, y aunque son considerables, concreta que habría que tratar las bibliotecas y obras compuestas por materias. Propone relacionar la historia pasada con las diferentes materias de estudio.

De todos estos pensamientos, y como relata Punzano (1981), se comprueba que la bibliografía, frecuentemente menospreciada, no se tiene que basar únicamente en enumerar obras, sino que también tiene que tener su origen en la actividad intelectual. Por lo tanto, la personalidad e interpretación del bibliógrafo será esencial (Gallego, 2005). Así, la bibliografía tiene que traspasar el ámbito técnico para convertirse en un campo amplio y crítico, como ya hacían diversos tratados de los siglos XV y XVI. Punzano (1981: 155) ya relata que estas obras tenían "un nivel muy superior que el de la mejor lista de las meramente técnicas". Y de la misma forma que opina Menéndez Pelayo (1876), la bibliografía tiene que servir -en palabras de Punzano (1981) y como piensan y detallan Escudero y Perosso (1894), Zarco del Valle y Sancho Rayón (1863) - para facilitar el trabajo intelectual y además para "mostrar la historia científica y literaria de un pueblo" (Punzano, 1981: 155). En esta línea, el discípulo de Menéndez Pelayo, Rufino Blanco (1907), también es de la 
opinión que hay que conocer los materiales recolectados de forma reflexiva para comprobar los efectos que han producido en un pueblo. Para este autor también es básico el uso de la bibliografía por materias, herramienta auxiliar de la filosofía y la historia, ya que a partir del análisis de las obras se puede conocer y reflexionar sobre la expansión de las ideas que promueven y las influencias que originan (Blanco, 1907).

Acorde con estas ideas, el objetivo del trabajo ha sido la elaboración de un repertorio bio-bibliográfico ajedrecístico español, de c. 1770-1899. Esta franja de estudio se justifica por las características políticas y sociales del siglo XIX que fomentan las publicaciones de libros. A este repertorio se le ha añadido un análisis crítico de las obras consultadas, además de aportar informaciones biográficas de los autores y contextualizar cada obra en la sociedad del momento. A todo ello hay que añadirle los análisis estadísticos, enmarcados en la bibliometría histórica (Hérubel, 1999), en relación con la producción de los libros en los diferentes años y lugares, la cantidad de obras originales españolas y el contenido-temática de las obras. El estudio viene acompañado de una contextualización de las obras en la época y finaliza con un estudio bibliométrico.

\section{LOS REPERTORIOS BIO-BIBLIOGRÁFICOS}

Los repertorios bio-bibliográficos son elementos de conocimiento sobre la evolución histórica a la vez que favorecen las diferentes ramas del saber y ayudan al progreso de la sociedad (Brasó, 2016).

Díez (2000) clasifica las bio-bibliografías según el autor y subclasifica según diferentes aspectos. Opina además que estas obras recuperan una información relacionada con la cultura de una zona y sus personajes ilustres. De hecho, este doble análisis sólo se encuentra muy detallado en algunas pocas obras, como en el repertorio de Juan Catalina, premiado en 1897 por la Biblioteca Nacional, o el de Rafael Ramírez, también premiado, en 1916. Igualmente, Díez (2000) destaca la obra -también premiada por la Biblioteca Nacional, en 1932- de Agustín Millares y Manuel Hernández. La autora considera, juntamente con Caballero (2008) y Martínez (1993-1995), que el gran avance y perfección metodológica de este tipo de trabajos aparece en Simón Díaz (1994), a quien se le considera uno de los más importantes investigadores en este campo, y que como Menéndez Pelayo (1876) postula que debería haber un índice bibliográfico exhaustivo por cada materia.

En relación con las partes esenciales de estos estudios bibliográficos y bio-bibliográficos, son explicados detalladamente por Díez (2000). Tales 
partes son la búsqueda de información, la identificación y el análisis del material bibliográfico, y la descripción bibliográfica. A todo esto hay que añadirle todas las dificultades metodológicas, que son entre otras la escasez de noticias y estudios, la lejanía de las bibliotecas de consulta o la falta de recursos para la búsqueda (Díez, 2000; Pons, 1898).

En el caso que nos ocupa, la justificación sobre la elaboración de un repertorio viene dada entre otros motivos por la necesidad de no caer en el olvido de las ideas y, en definitiva, la cultura de nuestros antepasados. Cumple también el trabajo la función de poder ser un instrumento de búsqueda para futuros investigadores (Herrero, 1996).

\section{Los libros de AJEDREZ EN EsPaÑA, DE I770 A I899}

El ajedrez se benefició de la invención de la imprenta. Inicialmente las obras iban destinadas a la clase alta, pero posteriormente, gracias a la disminución de los costes de los libros y simplicidad de sus contenidos, se amplió el campo a la población de menor estatus social (Brasó, 2014, 2016; Lasker, 1920; Ricart, 1897).

Aunque en España ya aparece el manuscrito de Alfonso X el Sabio en 1283, y posteriormente otros como los de Cesolis o el famoso poema Scachs d'Amor (Calvo, 1999), no es sino hasta 1495 que hay referencias del primer libro sobre el tema: el Llibre dels jochs partitis dels schachs, publicado en Valencia por Francesch Vicent -actualmente sin ningún ejemplar conocido en el mundo-. Posteriormente, aparecen otras obras como el Dechado de la vida Humana. Moralmente sacado del juego del Axedrez -publicado el 1544 en Salamanca-, el Libro de la invención liberal y arte del juego del Axedrez, de Ruy López -Alcalá, 1561-, o la obra desaparecida de Alfonso Cerdán, Del juego del axedrez, publicada alrededor de 1570 (Garzón, Alió y Artigas, 2012). Pero los inicios de la producción de los libros, y por tanto los inicios de la divulgación del ajedrez en España, surgen a finales del siglo XVIII e inicios del XIX. La primera obra y que marcaría el punto inicial es la de Pablo Minguet Irol -c. 1770- [1]. ${ }^{1}$ Su aparición coincide con los cambios científicos y culturales de la segunda mitad del siglo XVIII, que entre otros aspectos promulgaban una promoción de la cultura (Sobrequés, 1966; Vicens Vives, 1942).

Asimismo, es de relevancia el Compendio de los elementos teóricos y prácticos del juego del agedrez, publicado en París el año 1810 -1817- [2]. Este "es el primer libro impreso en España desde el siglo XVI en el que, además de

1 Se ha incluido en cada cita el número, entre corchetes, que ocupa en este repertorio. 
las reglas de juego, se incluyen partidas y finales" (Garzón, Alió y Artigas, 2012: 214).

De todas formas fue hasta la década de 1840 que apareció la primera obra de alto nivel ajedrecístico, traducida a multitud de idiomas. Fue el tratado de La Bourdonnais -1843- [8]. Su autor fue, además de mejor jugador del mundo, editor de la revisa La Palamède -1836-1839-. "Constituye la primera obra relevante desde el punto de vista ajedrecístico impresa en lengua castellana desde el s. XVI" (Garzón, Alió y Artigas, 2012: 230). Esto indica el creciente interés por el ajedrez en España a mediados de siglo XIX, después de un periodo en el cual tiene poca importancia en la sociedad. Posteriormente apareció la obra El ajedrez. Reglas del mismo y tratado para aprender a jugarle -1853- [12], un compendio reducido y en rústica de la obra de 1843 de La Bourdonnais, y que coincide con su segunda edición. Este hecho es un elemento más que refleja el crecimiento en la divulgación del juego, con una obra de coste económico menor, y por lo tanto más accesible. Este afán de divulgación es continuo y en 1864 aparece en Palma de Mallorca el Juego del ajedrez, sus reglas y leyes [14], de tan sólo 16 páginas y de pequeñas dimensiones, con el objetivo de ser un manual básico para iniciados. Estos hechos se relacionan con las primeras sociedades ajedrecísticas, de las cuales se encuentran los primeros indicios en el Juego del ajedrez, de 1838 [5]. Parece que los inicios del asociacionismo en ajedrez se citan en torno a este periodo en Barcelona (Brasó, 2014; Caballé, 1941).

Un hecho ligado a esta introducción social del ajedrez se aprecia en la aparición del juego en la prensa (Brasó, 2014) y también en la creación de un conjunto de obras literarias que utilizan el ajedrez entre sus páginas. Son destacables así la novela traducida del francés por Anónimo -1845- [9], la obra teatral de García Muñoz -1850- [10], la de Al Margheritíy -1872- [15], o la comedia de González de Iribarren -1878- [16]. Las obras no aportan información relevante del juego pero el ajedrez aparece en el ámbito literario, hecho que evidenciaría la curiosidad que despertaba en la sociedad española de la época.

Este afán de divulgación se combina en la década de 1840 con las primeras obras técnicamente más elaboradas, hecho que da un impulso al juego; si bien se trata de traducciones. Destacamos la traducción del ya citado La Bourdonnais -1843- [8] y la de la obra de Filidor -1880- [17]. Es en 1858 cuando se redacta la primera obra de calidad por un autor español: Gerónimo Borao [13].

Estos inicios literarios y asociacionistas se relacionan con la aparición de otras obras técnicas y manuales como la Introducción al noble juego de ajedrez -1887-1888- [18], y nuevas ediciones de obras ya impresas como la de 
Filidor, Borao o Clemente. Este interés ajedrecístico se materializa con una de las más interesantes obras de la historia del juego, el tratado de Brunet y Bellet -1891- [19]. La repercusión nacional e internacional en su época ("Bibliografía”, 1891; Garzón, Alió y Artigas, 2012; Tolosa, 1892) y la multitud de ediciones que se han hecho muestran su impacto y calidad. La hipótesis del autor es la de suponer que el ajedrez tiene su origen egipcio, a diferencia de anteriores opiniones.

Un año después a la obra de Brunet aparece la de Tolosa -1892- [20], publicada en Francia por Preti, director de La Stratégie. El objetivo del autor es tener repercusión internacional. Tolosa pensó que su publicación en España implicaría una baja repercusión en el extranjero, teniendo en cuenta la poca consideración general que se tenía por el juego (Brasó, 2014). El tratado se ha incluido en el repertorio bibliográfico y es la única excepción, ya que la repercusión del escrito en España es muy significativa, además de la nacionalidad catalana del autor. Parece que la idea de Tolosa fue un acierto. El tratado se anunció y se publicitó, además de en España, en Francia y en otros países, como en Cuba. En este caso apareció en la revista Capablanca-Magazine. El libro además se resumió en posteriores obras y prensa escrita, como en La Stratégie, en el libro ABC des echecs (Preti, 1895) y en la parte quinta del Manual de Ajedrez de José Paluzíe (Garzón, Alió y Artigas, 2012).

Posteriormente aparecen en 1896 dos obras de Márquez Sterling, Páginas de ajedrez-1896- [22] y Tratado analítico del juego de Ajedrez-1896- [23]. Se pueden comprobar la multitud de influencias extranjeras que había en España, tanto de autores de habla castellana como extranjeros. La relación con Clemente es más que evidente. Además se anuncia la que será una de las revistas específicas de ajedrez, dirigida por el autor: la Revista Internacional de Ajedrez-1896-.

Pero, seguramente, una de las obras más influyentes en España es el tratado divulgativo de Martín Ricart -1897- [24]. El manual técnico es sencillo y de pocas páginas. La cantidad de ediciones muestra la relevancia de la obra durante todo el siglo XX.

Martín, en la misma línea que Brunet y Bellet, hace una pequeña aportación histórica afirmando el origen persa del juego y contradiciendo muchas voces que ubicaban en los árabes el momento inicial del ajedrez. A la vez niega la práctica del juego en los griegos y romanos. Las aportaciones históricas se juntan con los diferentes motes que tuvo el juego en las diferentes culturas. El autor cita además la obra de Vicent como la primera publicada en Europa, y quemada -el último ejemplar- en 1835 a causa de la destrucción de los conventos. También cita Martín la obra de Lucena Repetición de amores y arte de Axedres con C.L. juegos de partido, de 1496, por Leonardo Hutz y Lope Sanz. 
Detalla que sólo quedan dos ejemplares. Asimismo, cita el Tratado de Damiano y el Libro de Ajedrez de Brunsvick, duque de Luxemburgo.

A la obra de Martín siguió la de Capó González -1899- [25], director de la publicación periódica Ruy López -1896-1899-. La obra tiene su origen en la revista, donde empezaron a aparecer los contenidos que posteriormente se publicarían en formato de libro. Es destacable la parte histórica, con informaciones de Brunet y Borao, y mención a las primeras obras españolas (Garzón, Alió y Artigas, 2012).

En el mismo año se publica, también en Barcelona, el tratado de Pin y Soler -1899- [26], en catalán. El autor lo dedica a Brunet. Pin y Soler hace un repaso histórico relacionado con el ajedrez, tanto por lo que respecta a libros - destacando, por cierto la obra desaparecida de Vicent y el tratado de Tolosa-, como al juego o a las leyes en Cataluña. La publicación, únicamente en catalán, tiene un sentido patriótico, lo que le lleva a criticar la revista Ruy López, editada por catalanes aunque escrita en castellano. La obra detalla problemas y explica diferentes soluciones de ajedrecistas bien conocidos en éste ámbito, como Marín, Paluzíe o Bosch de la Trinxería, entre otros.

Pero el afán por divulgar el ajedrez ya se inicia antes de la mitad del siglo XIX en obras que abarcan multitud de juegos. Es el caso de la Colección general de juegos permitidos para la distracción de toda clase y condición de la sociedad-1839- [6]. Se deduce así la influencia y relevancia social del ajedrez. Además, se comprueba la influencia de las máximas potencias extranjeras en materia ajedrecística. En este caso, se comenta que la parte de ajedrez se ha elaborado partiendo de la obra de ajedrez parisiense y traducida por Melchor de Sas -1817- [2]. De forma similar aparece la obra Tesoro de Juegos de Sociedad-1851- [11], de la cual se hicieron numerosas ediciones entre las cuales destaca la de 1881, corregida y aumentada. A finales del siglo XIX aparece el volumen de Marco y De Ochoa -1895- [21] y que parece que tuvo una segunda edición un año después. El tratado, de más de 1000 páginas, detalla en la parte correspondiente al ajedrez el juego, su reglamento, la historia, un diccionario, además de incluir partidas. El libro no aporta ninguna novedad a los escritos anteriormente.

Otro campo a tratar y que se visualiza en diferentes obras es la relación del ajedrez con la guerra, principalmente en las obras anteriores a la década de los años 30 del siglo XIX. Se encuentra el Diccionario del juego del ajedrez, ó ya sea vocabulario -1825- [3], que aunque describe las piezas, movimientos y ejemplificación de partidas, tiene un sentido bélico, ya que el autor explica que publica la obra para que se pueda entender la que posteriormente se publicará: El Juego de estratografía: ó Ajedrez-militar y escuela práctica de generales -1827- [4]. En la obra ajedrecística el autor adapta el ajedrez teniendo en 
cuenta las diferentes propuestas anteriores -como la de la Gaceta de Madrid, en junio de 1807-, para que sea transferible a la táctica y estrategia de la guerra. En este juego aparecen muchos más escaques.

Se comprueba así que la difusión de las obras de ajedrez del periodo analizado abarca diferentes campos, como la guerra, los juegos, la literatura o la historia.

\section{MÉTODO, ORDENACIÓN Y CRITERIOS DE SELECCIÓN DE LAS OBRAS}

Se consideran las obras de ajedrez susceptibles de análisis publicadas en España, por autores autóctonos o extranjeros. ${ }^{2}$ Los libros se han agrupado en tres tipologías: prácticas, teóricas y literarias. Las obras prácticas las conforman manuales técnicos (MT), ajedrez y otros juegos (JU), partidas (PA), problemas (PR), reglamento (RG). Las teóricas se dividen en biografías (BI), diccionarios (DI), históricos (HI). Finalmente se han añadido las obras literarias (LT), que incluyen novelas, poemas y obras teatrales relacionados con el juego. Aunque para cada libro se indica de forma específica su contenido, para el posterior análisis estadístico se han tenido en cuenta de modo global las tres tipologías iniciales citadas.

Para su elaboración se ha seguido la metodología que propone Simón Díaz, descrita por Díez (2000). Han sido analizados los repertorios ajedrecísticos publicados hasta la fecha. Es relevante remarcar que el bajo número de éstos, como sucede normalmente, muestra la desproporción que hay en España entre el número de obras publicadas y sus repertorios (Torres, 1992).

Por lo que respecta a las publicaciones fuera de España, se encuentran diferentes tratados (Clemente, 1899 [obra facilitada por la biblioteca de Princeston]; Di Felice, 2010; Simpson, 1863). En relación con la aportación bibliográfica ajedrecística española se han analizado diferentes obras, manuales y catálogos (Brasó, 2013; Fe, 1903; Garzón, Alió y Artigas, 2012; Índice Bibliográfico, 1933; Índice Bibliográfico de Obras de fondo y algunas de surtido, 1912; Llaverías, 1910; Paluzíe, 1943, 1921, 1912; Sánchez, 1934). Finalmente, los catálogos de las diferentes bibliotecas españolas, algunos de ellos digitalizados, han concluido esta búsqueda. Destacan las bases de datos de la Biblioteca Nacional de España (BNE), de la Red de Bibliotecas Universitarias (REBIUN), de las Bibliotecas Públicas del Estado (BPE), o de la Biblioteca de Cataluña (BC).

2 Se ha incluido una excepción: es la obra del catalán Tolosa (1892), que tuvo alta repercusión en España. 
De cada asiento bibliográfico consignado en el repertorio se detallan las siguientes informaciones: ${ }^{3}$

[Número de la obra en el repertorio] Año; AUTOR ${ }^{4}$ [NOMBRE COMPLETO, AÑO Y FECHA DE NACIMIENTO Y DEFUNCIÓN]: Titulo de la obra completo. Localidad: editorial, imprenta, entidad que publica la obra, año. [Descripción física $\left.^{6}\right]$ Tipología del contenido en las obras.

Juntamente con la bibliografía, en el campo de la bibliometría histórica se analizan diferentes variables de estas fuentes -libros- primarias. Como consecuencia, se muestra un conjunto de tablas y datos estadísticos, elementos indispensables en este tipo de trabajos (Pons, 1898). El análisis se refiere a la producción según la época, localidad de edición, la traducción u originalidad de la obra, y su tipología. A la vez, en la misma línea que Menéndez Pelayo (1876), el repertorio trata el contenido bibliográfico y su tratamiento estadístico, al tiempo que reflexiona sobre las mismas obras en su época, hecho detallado en el punto anterior.

\section{REPERTORIO DE LAS OBRAS}

[1] 1770; MINGUET IROL, PABLO [c.1700/1715-1780/1801] : Origen y reglas del ingenioso juego del axedrez / Obra muy util y provechosa tanto para los que de nuevo quisieren aprenderlo, como para refrescar la memoria de los que ya saben jugarlo. Su autor__, Grabador de Sellos, Láminas, Firmas, y otras cosas. ${ }^{8}$ Barcelona: Oficina de Carlos Gibert y Tutó, administrada por Antonio Sastres, 1770. ${ }^{9}$ [16 p.: 15 $\mathrm{cm}$.] HI-RG

3 Se ha adaptado y simplificado la descripción con base en la International Standard Bibliographic Description (ISBD), redactada por la Federación Internacional de Asociaciones de Bibliotecarios y Bibliotecas (FIAB). Es notorio destacar que se ha utilizado una descripción de las obras en la línea de Torrebadella (2011).

4 Se ha añadido información adicional: nombre completo del autor, año y lugar de nacimiento y defunción.

5 Mediante la barra [/] se indica el fin de línea en el título original de la obra.

6 Número de páginas, ilustraciones y altura de la cubierta en centímetros.

7 Pons (1898).

8 Anteriormente el autor publicó tratados de damas, naipes y chaquete. Se ve el carácter divulgativo en el hecho que se comenta que para el juego hacen falta simplemente botones. En venta en la librería de Palau (1925) - nº 27, ref. 305, p. 14-. La librería disponía de multitud de obras ajedrecísticas, y también de la revista mensual de Capó, Ruy López, en 4 tomos (Barcelona, 1896-99), a 50 pesetas.

9 Hay otra edición de la obra en 1796 (Garzón, Alió y Artigas, 2012). 
[2] 1817; D. M. de S. [Melchor de Sas]: ${ }^{10}$ Compendio de los elementos teóricos y prácticos del juego del agedrez, publicado en París el año 1810 / Traducidos y compendiados por___. Barcelona: Imprenta de Tomas Gorchs, ${ }^{11}$ 1817. ${ }^{12}$ [85 p.: ${ }^{13}$ il.: con 1 dibujo $;{ }^{14} 15 \mathrm{~cm}$.] MT

[3] 1825; E. Y. D. J. D. L. T. C. Y. A. L. [Yngeniero Don Joaquín De La Torre $^{15}$ ]: Diccionario del juego del ajedrez, ó ya sea vocabulario / De las voces técnicas, pertenecientes y mas en uso en este Juego. Con sus definiciones, y esplicaciones, entre las cuales van comprendidas sus Reglas y Leyes con egemplos prácticos, sencillos, instructivos y curiosos, para aprenderlo sin la ayuda de Maestro; por que van demostrados al alcance de todos. Obra única en su clase y método. Dispuesta y ordenada para facilitar su inteligencia por el autor del Juego ortográfico de la palabra de la lengua castellana. ${ }^{16}$ Barcelona: Imp. de José Torner, ${ }^{17} 1825$. [55 p.: ${ }^{18} 22 \mathrm{~cm}$.] DC

[4] 1827; E.I.D.J.D.L.T.-C.Y.A. -L. [Ingeniero Don Joaquín De La Torre ${ }^{19}$ ]: El Juego de estratografía: ó Ajedrez-militar y escuela práctica de genera$\operatorname{les}^{20} /$ Traducido del alemán al francés y de este al castellano sobre la segunda edición y adornado con reflexiones morales políticas históricas y militares muy propias del espiritu del juego. Por el autor del juego ortográfico de la palabra ect_-_21. Barcelona: Imp. De Viuda é hijos de D. Antonio Brusi, 1827. [78 p.: 2 láminas con dibujos y tablero; $20 \mathrm{~cm}$.] JU

[5] 1838; ANÓNIMO: Juego del ajedrez. Madrid: Imp. de Yenes, ${ }^{22} 1838$. [28 p.: $16 \mathrm{~cm}]$ MT

10 El autor es el francés L. Hocquart (Paluzíe, 1912, 1921, 1943) y Palau (1923-1945)-ref. 115461-.

11 Dirección: Bajada de la cárcel. En la portada del libro se aprecia el símbolo de la editorial.

12 2a. ed. por la imp. de Viuda e hijos de Gorchs (1838) y facsímil -París-Valencia, 1998-. Se publicó en Barcelona, un extracto de la obra (1839). P. F. y P. es Don Juan Francisco Piferrer Impresor de S. M. en la plaza del Ángel-. La obra aparece en el catálogo de la librería de Palau (1925) $-n^{\circ} 27$, ref. $267-$.

13 Hay dos páginas finales sin numerar, que incluyen una lámina y una errata en la p. 56.

14 Lámina de las 16 piezas del ajedrez.

15 Paluzíe $(1912,1921,1943)$ y Palau (1923-1945). Aunque según Pastor (1995) el original es de Hocquart (1810) -ref. 7539-.

16 Incluye una cita de una parte del Poema del Ajedrez por el Abad Roman. Se citan diferentes obras de ajedrez que tienen que publicarse. El autor publicó la obra Escuela-Práctica de Generales.

17 Calle de Capellans. La obra se vendía en la librería de Palau (1925)-nº 27, 1925, ref. 269-.

18 Hay cuatro hojas plegables - tableros y tabla de anotación- y una final con información adicional.

19 Paluzíe (1912, 1921, 1943) y Palau (1923-1945). El original según Pastor (1995) es Hocquart.

20 Se comenta refiriéndose al título que "El juego es propiamente una Escuela práctica para los aspirantes a Generales en el lleno de todas sus obligaciones en campaña, sin que le falte circunstancia".

21 El traductor se ha basado en un artículo aparecido en La Gaceta de Madrid (junio de 1807).

22 Calle de la Almudena. El libro se hallará en la librería de Cuesta, frente a las Covachuelas. 
[6] 1839; D. P. F. y P.: ${ }^{23}$ Colección general de juegos permitidos para la distracción de toda clase y condición de la sociedad / Por___24. Barcelona: Don Juan Francisco Piferrer, ${ }^{25}$ Impresor de S.M. Plaza del Ángelz, 1839. [403p.: ${ }^{26}$ il. con dos diagramas; ${ }^{27} 15 \mathrm{~cm}$.] JU

[7] 1839; P. F. Y P.: Compendio de los elementos teóricos y prácticos del juego del ajedrez / Por___. Barcelona: Don Juan Francisco Piferrer, Impresor de S.M. Plaza del Ángel, $1839 .{ }^{28}$ [49 p.; $16 \mathrm{~cm}$.] MT

[8] 1843; BOURDONNAIS, Mr. L. C. de la [LOUIS CHARLES MAHÉ. (BOURBOUN), 1795/7-(LONDRES), 1840]: Nuevo tratado del juego del Agedrez. / Por___. Traducido del Francés. Cádiz: Imp., librería y litografía de la Revista Médica, ${ }^{29} 1843 .{ }^{30}\left[366\right.$ p. ${ }^{31}$ : con 61 diagramas y un grabado $\left.{ }^{32} ; 22 \mathrm{~cm}\right] \mathrm{MT}$

[9] 1845; Anónimo: Una partida de ajedrez / Novela escrita en francés por___ y traducida al castellano por J. M. P. Cartagena: Imp. de Nicolas Nadal, $1845 . .^{33}$ [46 p.: $15 \mathrm{~cm}$ ] LI

[10] 1850; GARCÍA MUÑOZ, MANUEL: El juego de ajedrez, comedia en cuatro actos / Joyas del Teatro. Colección de las mejores obras dramáticas representadas en todos los teatros de España y ultramar. ${ }^{34}$ Barcelona: Impr. Y Libr. Viuda e Hijos de Mayol Editores, ${ }^{35}$ 1850. [20 p.: $26 \mathrm{~cm}$ ] LI

[11] 1851; ANÓNIMO: ${ }^{36}$ Tesoro de Juegos de Sociedad / Contiene las reglas y leyes de más de treinta juegos permitidos en toda clase de sociedades, casinos, reuniones particulares y cafés. El Tresillo. Billar. Ajedrez. Whist. Dómino. Lotería. Chaquete. Damas. Solo. Ciudadela. Malilla. Batalla. Ecarté. Báciga. Tercera edición corregida y aumentada. Barcelona: Manuel Saurí, $1851 .^{37}\left[316\right.$ p. $\left.;^{38} 15 \mathrm{~cm}\right] \mathrm{JU}$

23 Podrían ser las iniciales de Francisco Piferrer.

24 Resumen de Compendio de los elementos teóricos y prácticos del juego del ajedrez (1838, 2a. ed.).

25 Propiedad de la casa Piferrer. Se detalla la notación: "impresor de S. M. Plaza del Ángel".

26 En cada capítulo empieza la numeración.

27 Sólo se ha tenido en cuenta el capítulo referente al ajedrez.

28 Extracto del Compendio de elementos teóricos y prácticos del juego del ajedrez... (Barcelona, 1817). Hay una edición de 1851, aunque en este caso el editor es José Piferrer. Además, el impresor publica la Colección general de juegos permitidos para la distracción de toda clase y condición de la sociedad, con la parte sobre ajedrez idéntica en los dos libros (Garzón, Alió y Artigas, 2012).

29 Plaza de la Constitución, $n^{\circ}$ 11. La ed. de 1853 es a cargo de D. Juan Bautista de Gaona.

30 Original en París (1833). 2a. ed., 1853; facsímil por París-Valencia (1998), y Nabu Press (2012).

31 Se divide en dos libros (167 p.; 199 p.).

32 Grabado en la portada: un caballero y una dama jugando al ajedrez y dos caballeros mirando.

33 Noviembre.

$34 \mathrm{~N}^{\circ} 43$.

35 Calle de Fernando VII, n $^{\circ} 29$.

36 El autor podría ser Vindel.

37 Otras ed. aumentadas y corregidas: 2a. -Saurí y Sabater. Imp. Jaime Jepús- (1875); 3a. -Manuel Saurí- (1881); 4a. -Manuel Saurí- (1887); 5a. (1896); 6a. -Hijos de Francisco Sabater(1911); 7a. -Puig- (1934). 
[12] 1853; LA BOURDONNAIS, L. C. [LOUIS CHARLES MAHÉ. BOURBOUN, 1795/7-LONDRES]: El ajedrez. Reglas del mismo y tratado para aprender a jugarle / Salida de Arfil-Salida de caballo-Gambito de Rey-Gambito de Reina-Salidas variadas-Partidas con ventaja-Finales de juego-Mates-Posiciones curiosas. Extractado todo de por un aficionado. ${ }^{39}$ Madrid: Imp. Que fue de Operarios, a cargo de D.F.R. Del Castillo, ${ }^{40} 1853$. [89 p.: con 6 diagramas; $19 \mathrm{~cm}$ ] MT-RG

[13] 1858; BORAO, GERÓNIMO [CLEMENTE, ZARAGOZA, 18211878]:41 El ajedrez. Tratado de sus principios fundamentales / Por D.___. Precédele una Introducción histórica, y síguele algunas partidas de Philidor y otros, y un poema de vida traducido espresamente para esta obra por Ángel Gallifa. ${ }^{42}$ Zaragoza: Lib. de V. Andrés, ${ }^{43}$ $1858 .{ }^{44}[161$ p.; $21 \mathrm{~cm}]$ DI-HI ${ }^{45}$-LI-MT

[14] 1864; ANÓNIMO: Juego del ajedrez, sus reglas y leyes. Palma: Impr. de A. Haro, 1864. [16 p.: con 1 diagrama; $11 \mathrm{~cm}^{46}$ ] MT

[15] 1872; AL MARGHERITÍY: Un juego de ajedrez: leyenda árabe-granadina (810-H.--1408-J.C.) ${ }^{47}$. Madrid: Imp. de T. Fortanet, 1872. [82 p.; $17 \mathrm{~cm}] \mathrm{LI}$

[16] 1878; GONZÁLEZ DE IRIBARREN, JOSÉ: La partida de ajedrez / comedia en un acto y en verso original de ___. Estrenada con extraordinario aplauso en el Teatro de la Comedia el 13 de Marzo de 1878. Madrid: Imp. De José Rodríguez, ${ }^{48} 1878 .{ }^{49}$ [28 p.; 20 cm] LI

[17] 1880; FILIDOR, A. D. [FRANÇOIS-ANDRE DANICAN. DREUX (FRANCIA), 1726-1795]: Análisis del Juego de Ajedrez / Por_._. Con una nueva anotación compendiada y láminas en las cuales está figurada la situación del juego para las llamadas y conclusión de partidas. Nueva edición aumentada con un tratado de estratagemas. Traducción de D.

39 Resumen-copia del Nuevo tratado de Ajedrez, de 1843 (Garzón, Alió y Artigas, 2012).

40 Calle del Factor núm. 9.

41 El autor era aficionado. Justifica la obra teniendo en cuenta la aportación didáctica, inexistente en las anteriores publicadas y su entusiasmo. Da a conocer un poema de Gallifa.

42 Obra de calidad, donde hay una bibliografía ajedrecística, de libros y de publicaciones periódicas, y se cita por primera vez la obra de Francesch Vicent -Valencia, 1493- (Garzón, Alió y Artigas, 2012).

43 Calle Cuchillería, 42. Se indica también: Biblioteca de "El Saldubense".

44 Tip. Julián Sanz y Navarro (Hijos de Uriarte), calle de Alfonso I, n 20. Reimpresión en 1859; 2a. ed. en 1888; 3a. ed. en 1901; facsímil por París-Valencia (2004). Libro anunciado incluso en prensa no ajedrecística, como en La Esquella de la torratxa (28 abril 1888, n. 485, p. 14).

45 Aparecen multitud de variantes ajedrecísticas practicadas en diferentes países.

46 Libro de pequeñas dimensiones. En el ejemplar de la Biblioteca de Cataluña hay una nota a mano donde se informa que parte de la biblioteca de ajedrez de Tolosa pasó a Paluzíe -Barcelona, 1916-.

47 Se hacen aportaciones lingüísticas y evolutivas del nombre de las piezas.

48 Calle Calvario, 18.

49 Hay una 2a. ed. en 1890, de la Imp. Jose Rodríguez. 
C. de Algarra. Ludimuseffigiem belli. Vida. ${ }^{50}$ Madrid: Librerías de A. de San Martín, ${ }^{51}$ c. $1880^{52}$ [301 p.: il. con 60 diagramas; 19 cm.] MT

[18] 1887-1888; ANÓNIMO [DE EPALZA, JOSÉ (BILBAO, 1854-1915)]: Introducción al noble juego de ajedrez. ${ }^{53}$ Bilbao: Tip. Cat. de San Francisco de Sales, ${ }^{54}$ Lib. De Bulfy y Ca., 1887-1888. $\left[2 v^{55}\left(48 \mathrm{p}^{56}+158\right.\right.$ p. ${ }^{57}$ ): con diagramas; $12 \mathrm{~cm}^{58}$ ] MT

[19] 1891; BRUNET Y BELLET, JOSÉ [BARCELONA, 1818-1905]: El ajedrez: investigaciones sobre su origen / Por ___. ${ }^{59}$ Barcelona: L'Avens ${ }^{60}$ Lib., $1891 .{ }^{61}$ [426 p.: ${ }^{62}$ il. con 95 dibujos; ${ }^{63} 26 \mathrm{~cm}$ ] HI

[20] 1892; TOLOSA CARRERAS [JOSÉ. GIRONA, 1846-BARCELONA, 1916]: Traité analytique du problème d'échecs / Complété par un exposé de tous les genres de problèmes, quelques réflexions critiques sur les concours et un recueil de problèmes d'auteurs espagnols. Par le Dr.__.. París: Numa Preti, ${ }^{64}$ 1892. [446 p.; 18 cm] PR

50 Se trata de Don Carlos de Algarra (Garzón, Alió y Artigas, 2012). El autor fue traductor en su exilio de diferentes tratados: La Educación física de los niños, ó Consejos á las madres sobre el amamantamiento y la manera de criar a sus hijos. Ver Torrebadella (2009).

51 Puerta del Sol, 6, y Carretas, 39. El Libro de Oro.

52 Original francesa de 1749. En 1750 se tradujo al inglés, el 1754 al alemán, y el 1777 se publica en Londres. La 1a. ed. española es en Méjico (1846) por el mismo traductor -n ${ }^{\circ}$ 62- (Paluzíe, 1943). Por lo tanto era conocida en España. También se publicó en Buenos Aires, por Sopena Argentina (1952). La primera parte de la obra es una reimpresión corregida y aumentada del original de 1749. La primera traducción de la obra fue en Madrid el 1827 -ms. 6414- y se encuentra en la BNE. Hay una 2a. ed. española c. 1890. Además, hay multitud de ediciones, numerosas anteriores de la impresión en España de la obra: Lib. de Rosa y Bouret en París (1846 -tip. De Lacrampe y comp., calle Darniete-, 1850, 1857, 1864 -Tip. Lacrampe y com y otra de tip. y ster. De Aug. Bouret-, 1870 -Simon Bacon y comp.-), Lib. C. Bouret en París / Méjico (1877 -imp. Julio le Clere y comp.-, 1881 -imp. F. Levé-), Imp. Gobierno Federal en Méjico (1885), Lib. Ch. Bouret en París / Méjico $(1887,1891)$, Lib. de la Vda de Ch. Bouret en París / Méjico (1898, 1901, 1906, 1911, 1919, 1922, 1926), Lib. Vda de Ch. Bouret en París (1929, 1936). Hay una 7a. ed. en 1978. Hay facsímiles por Paris-Valencia (1992) y Maxtor (2004).

53 Subtítulo de la primera parte: Salidas modelo y reglas de juego. Segunda parte: La Estrategia del tablero y las posiciones más correctas que pueden formarse en las cinco primeras jugadas. Precio: 1 peseta la docena de ejemplares, la primera parte, y 1 peseta el ejemplar, la segunda parte.

54 París á Mr. Preti; 74, Rue Saint Sauveur.

55 En la Biblioteca de Catalunya (BC) se encuentra la obra en uno y en dos tomos.

56 Hay tres diagramas en la primera parte.

57 Página inicial + 158 p. +8 tableros vacíos e índice.

58 Tip. Cat. de San Francisco, en París á Mr. Preti; 74, Rue Saint Sauveur. En la portada de la primera parte hay una cita sobre la importancia de la salida. Precio por volumen: 1 peseta.

59 En la portada hay un grabado -también en la p. 397- con la reproducción del papirus de Turín. Representa a Ramsés III jugando con alguna de sus favoritas. También hay una cita de Leon Hugonnet. El autor dio el manuscrito y un ejemplar a la Biblioteca-Museu Balaguer (Donativo-Brunet, 1891).

60 Librería en Boters, 16. Est. tipo-litográfico de Espasa y Compañía. Se vendía en numerosas librerías, como en la de Palau (1925) -n 27 de 1925, ref. $263-$.

61 La obra se anuncia en L'Avenç (Bibliografía, 1891). Se vende a 15 pesetas. No empezó a circular hasta 1891 (Garzón, Alió y Artigas, 2012) a causa de la publicación de la obra en las revistas L'Avenç y La Stratégie -por Tolosa-. Hispano Europea (2005) y BiblioBazaar (2010), también la han publicado. En el ejemplar de la Biblioteca-Museo Víctor Balaguer, hay una dedicatoria del autor a la biblioteca.

62 Hay dos páginas al final de fe de erratas y una última página de obras del mismo autor.

63 Dibujos históricos: tableros chinos, piezas, escudos, papirus...

64 72, Rue Saint-Sauveur. 
[21] 1895; MARCO, DON LUIS; DE OCHOA, DON EUGENIO: ${ }^{65}$ Repertorio completo de todos los juegos / Juegos de naipes.- Juegos de puro azar.- Juegos de combinación.- Juegos de puro cálculo.- Juegos de precisión.- Juegos de sociedad.- Juegos de prendas.- Juegos de ingenio.- Los rompecabezas.- Entretenimientos matemáticos.- Física recreativa.- Juegos de niños en habitación cerrada.- Juegos de niños y niñas al aire libre.- El sport y juegos atléticos / recopilados por Don Ilustrado con más de 1000 figuras. Madrid: Casa editorial Bailly-Bailliere ${ }^{66}$ (ca. $1895^{67}$ ). [1014 p.: más de 1000 figuras; ${ }^{68} 26 \mathrm{~cm}$ ] JURG-HI-DC-PA.

[22] 1896; MÁRQUEZ STERLING, MANUEL [LIMA, 1872-WASHINTON, 1934]: Páginas de ajedrez. Con una carta-prólogo de A. C. Vázquez. / Con una carta-prólogo de Andrés Clemente Vázquez. Campeón de México. ${ }^{69}$ Madrid: Lib. de Fernando Fé, ${ }^{70} 1896 .^{71}$ [63 p.: il. con 2 fotos $^{72}$ y 5 diagramas; $\left.15 \mathrm{~cm}\right]^{73}$ BI-MT-PA-RG

[23] 1896; MÁRQUEZ STERLING, MANUEL [LIMA, 1872-WASHINGTON, 1934]: Tratado analítico del juego de Ajedrez: para aprender á jugarlo sin necesidad de maestro. Madrid: Est. Tip. de Ricardo Fé, ${ }^{74}$ $1896 .{ }^{75}$ [93 p.: con 1 foto $^{76}$ y 13 diagramas; $17 \mathrm{~cm}$ ] MT

[24] 1897; MARTÍN RICART ${ }^{77}$ [ARÍSTIDES MESTRES]: Manual del ajedrecista / obra utilísima no sólo á los principiantes si que también á los buenos jugadores por el sinnúmero de casos prácticos que contiene con abundancia de detalles rigurosamente históricos un capítulo especial de gambitos y contragambitos y una copia exacta de las grandes partidas que se han jugado en el presente siglo. ${ }^{78}$ Barcelona:

65 En relación con este segundo autor: Francia, 1815-Madrid, 1872.

66 Plaza de Santa Ana, nº 10. Agencia en Barcelona: Pelayo, n⿳ 8, entresuelo. En la cubierta posterior hay el símbolo de la editorial y hay escrito: L. Calleja, Encdor.

67 Según la BNE esta obra es de 1895. Hay una ed. de 1896.

68 Páginas relativas al ajedrez: 455-529. Con: 1 grabado, 51diagramas y 3 dibujos. El grabado es de ajedrecistas en el café de la Regencia, por G. Auprès Boilly y P. Grenier. sc.

69 Dedicado a Lasker.

70 C. San Jerónimo, 2. Se indica en la página anterior: Est. Tip. de Ricardo Fé, calle del Olmo, 4.

71 Se vende a 3 pesetas. Obra destacada en la Revista Internacional de Ajedrez (1896, p. 24) y en la Revista Contemporánea (15 de enero, 1896, pp. 108-109).

72 De S. Lipschütz -p. 26-y de H. N. Pillsbury-p. 42-.

73 Destaca la carta-prólogo -La Habana, 1895-, por Clemente y dirigida a Sterling -Nueva York-.

74 Calle San Jerónimo, 2.

75 2a. ed. aumentada (1897). Anunciada en: Revista Internacional de Ajedrez (1896, p. 55).

76 Del autor.

77 Pseudónimo.

78 En el Índice Bibliográfico (1933) -ref. 1094a y 1094b- cita que se vendía en dos partes, cada una a 3 pesetas, y se indica como autor a Puig erróneamente -(Paluzíe, 1912) - y no a Martín Ricart. 
Saurí y Sabater, ${ }^{79} 1897 .{ }^{80}\left[88\right.$ p.: ${ }^{81}$ il. con 6 dibujos y un diagrama; 20 $\mathrm{cm}$ ] MT-HI

[25] 1899; CAPÓ GONZÁLEZ, J. [PALMA DE MALLORCA, 1865-?]: La apertura española ó Ruy López / Estudio por___. Aperturas de ajedrez. Primer volumen. ${ }^{82}$ Barcelona: La Académica, ${ }^{83}$ 1899. [156 p.; $23 \mathrm{~cm}$ ] MT

[26] 1899; PIN Y SOLER, J. [JOSEP. 1842-1927]: Problemes d'escacbs d'autors catalans contemporanis / collegits y precedits d'un pròlech per J. Pin y Soler. ${ }^{84}$ Barcelona: Imp. De Henrich y Companyia, en comandita, $1899 .{ }^{85}$ [235 p..$^{86}$ il. con 11 dibujos y 137 diagramas. $\left.23 \mathrm{~cm}^{87}\right]$ PR

\section{ANÁLISIS ESTADÍSTICO DE LAS OBRAS}

En la producción de libros agrupados por el periodo de tiempo analizado cabe destacar que, de 1770 hasta 1824, la producción es mínima (8\%), y no es sino hasta el último cuarto de siglo que las publicaciones aumentan significativamente (42\%) (Tabla 1$)$.

Tabla 1. Distribución de las publicaciones de libros de ajedrez por periodos de años

\begin{tabular}{|c|c|c|}
\hline Periodo (años) & $\mathbf{N}^{0}$ obras & $\%$ \\
\hline C. 1770 a 1824 & 2 & 8 \\
\hline 1825 a 1849 & 7 & 27 \\
\hline 1850 a 1874 & 6 & 23 \\
\hline 1875 a 1899 & 11 & 42 \\
\hline Total & 26 & 100 \\
\hline
\end{tabular}

En relación con la localidad de impresión, Barcelona ocupa el primer puesto $(46 \%)$. Aparece así la primera publicación fuera de la ciudad condal, en 1838. Posteriormente a esta fecha, aunque la ciudad citada es también la principal en publicaciones, aparece Madrid ( $31 \%$ ) con un conjunto significativo de obras que se prolongará durante todo el siglo XIX. Aparte de estas

79 Calle del Notariado, núm. 9. Teléfono 151. Se indica también Imp. Jaime Jepús.

80 Ed.: 1913, 1921, 1925 y 1928 -por Francisco Puig-; 1955 -por Agustín Nuñez-, y facsímil por la Librería "Paris-Valencia” (1992). Hay una edición en México (1975).

81 Más ocho páginas en las cuales se citan diferentes libros de los editores Saurí y Sabater.

82 Los folletos de la revista Ruy López publicados por el autor, se editaron en formato de libro.

83 Serra hermanos y Russell, Ronda Universitat, 6. Teléfono 861.

84 Dedicado a Brunet. Hallase en la librería de Verdaguer, Rambla del Centro, 5. Barcelona.

85 Hay una reproducción de la obra de las editoriales NabuPress y Bibliobazaar (2010).

86 Sólo 35 páginas están numeradas. De las otras, hay 137 páginas de diagramas y 54 de soluciones. 
dos ciudades, aparecen otras localidades con sólo una obra y, por lo tanto, poco significativas (Tabla 2), que para el análisis estadístico se han agrupado en un apartado titulado "Otros" (23\%).

Tabla 2. Producción de libros ajedrecísticos según la localidad, de 1770 a 1899

\begin{tabular}{|c|c|c|}
\hline Lugar de edición & $\mathbf{N}^{\mathbf{0}}$ obras & $\%$ \\
\hline Barcelona & 12 & 46 \\
\hline Madrid & 8 & 31 \\
\hline Otros & 6 & 23 \\
\hline Total & 26 & 100 \\
\hline
\end{tabular}

Por lo que respecta a la producción española, hay que destacar la gran cantidad de obras que son traducciones completas o parciales de tratados extranjeros $(61.5 \%)$ en relación con las originales o de creación propia nacional $(34.5 \%)$ (Tabla 3$)$.

Tabla 3. Producción de libros españoles según sean o no traducciones

\begin{tabular}{|c|c|c|}
\hline Obras & No obras & \% \\
\hline Traducciones & 16 & 61.5 \\
\hline No traducciones & 9 & 34.5 \\
\hline Otras & 1 & 4 \\
\hline Total & 26 & 100 \\
\hline
\end{tabular}

En relación con la tipología de las obras (Tabla 4), el $23 \%$ tiene una temática teórica y un $19 \%$ literaria. Predomina así la cantidad de libros que, entre otras tipologías, tienen un carácter práctico (73\%). Es destacable además que el $50 \%$ de la totalidad son manuales técnicos, además de tratar otros contenidos.

Tabla 4. Cantidad de obras ajedrecísticas (1800-1899) según su contenido: teórico, práctico o literatura

\begin{tabular}{|c|c|c|c|c|c|c|}
\hline Contenido & Teórico & $\%$ & Práctico & $\%$ & Literatura & $\%$ \\
\hline Sí & 6 & 23 & 19 & 73 & 5 & 19 \\
\hline NO & 20 & 77 & 7 & 27 & 21 & 81 \\
\hline Total & 26 & 100 & 26 & 100 & 26 & 100 \\
\hline
\end{tabular}




\section{Discusión}

El conjunto de obras presentadas abarca todos los libros publicados en España desde finales del siglo XVIII hasta 1899, incluyendo como excepción justificada el tratado de José Tolosa de 1892. La primera obra del repertorio, de Minguet, simboliza el cambio social hacia la modernidad, en favor de la ciencia y la cultura, que surge en esta época (Sobrequés, 1966; Vicens Vives, 1942). Es el punto inicial del incremento significativo de publicaciones, hecho que favorecerá al conocimiento del ajedrez en las diferentes esferas sociales.

Por lo que respecta a las ciudades editoras de obras ajedrecísticas, destaca Barcelona, pionera también en muchos otros ámbitos. De todas formas, gran cantidad de las obras son traducciones, lo que muestra el retraso ajedrecístico, debido al retraso cultural, en relación con la mayoría de países europeos. Habrá que esperar a Borao y su tratado en 1858 para encontrar la primera obra de calidad producida en España. Se constata además que la mayoría de los tratados tienen un carácter práctico con un objetivo enfocado al aprendizaje para el lector -manuales, problemas, partidas-. Se comprueba que a medida que avanza el siglo XIX van apareciendo los primeros tratados de bajo coste.

\section{A MODO DE CONCLUSIÓN}

El estudio se ha limitado en el tiempo, hecho que hace necesarias más investigaciones relacionadas con la temática y así poder describir y relacionar el ajedrez con el contexto político, económico, social y cultural de cada época. En esta línea se ha pretendido ampliar las investigaciones en materia de ajedrez existentes ${ }^{88}$ además de proporcionar una base para futuros estudios. Por lo tanto, son necesarias mayores aportaciones históricas en materia de ajedrez que permitan entender y contextualizar el juego en la sociedad de cada época.

88 Concretamente el artículo es una continuación de un estudio publicado por el autor (Brasó, 2016). 


\section{REFERENCIAS}

“Bibliografía”. 1891. L'Avenç3 (5) (31 de mayo): 158-160.

Blanco, Rufino. 1927. Bibliografía general de la educación física: con datos copiosos referentes a la biología, antropología, anatomía, fisiología, higiene, eugénica, puericultura, paidología, pedagogía y otras ciencias fundamentales del ejercicio corporal educativo. Madrid: Hernando.

Blanco, Rufino. 1907. Biblioteca Pedagógica. Madrid: Tip. de la revista de Archivos, Bibliotecas y Museos, 1907-1912.

Bolado, Gerardo. 2012. "Menéndez Pelayo y las verdades de la tradición”. Eikasia 45: 251-262.

Brasó, Jordi. 2013. "Bibliografía de ajedrez existente en España, de 1800 a 1939". Lecturas: Educación Física y Deportes 18 (184). http://www.efdeportes.com/efd184/ bibliografia-de-ajedrez-en-espana-1800-1939.htm

Brasó, Jordi. 2016. "Los libros de ajedrez en España (1900-1939): Repertorio bio-bibliográfico y análisis bibliométrico”. Anales de Documentación 9 (1): 1-17. dx.doi. org/10.6018/analesdoc.19.1.247821

Brasó, Jordi. 2014. "Inicios del ajedrez en la prensa y en las publicaciones deportivas en España (1861-1915)”. Recorde: Revista de História do Esporte 7 (1): 1-34.

Caballé, Tomás. 1941. Evocaciones históricas barcelonesas. De cómo empezó en Barcelona... lo que verá el curioso lector. Barcelona: Fomento de la Producción Española.

Caballero, Manuel. 2008. Diccionario bio-bibliográfico del Santo Reino de Jaén. Madrid: CSIC.

Calvo, Ricardo. 1999. El poema Scachs d'amor. Madrid: Jaque XXI.

Clemente, Andrés. 1899. Biblioteca 'Andrés C. Vázquez'. Habana: Avisador Comercial.

Di Felice, Gino. 2010. Chess Periodicals. An Annotated International Bibliography, 1836-2008. North Carolina: McFarland \& Company.

Díez, Isabel Cristina. 2000. "Las bio-bibliografías: estado actual y metodología”. Cuadernos de documentación multimedia 10: 67-77.

Donativo-Brunet. 1891. Revista Biblioteca-Museu Balaguer 78: 2-3.

Escudero y Perosso, Francisco. 1894. Tipografía hispalense. Madrid: Suc. de Rivadeneyra.

Fe, Fernando. 1903. Catálogo de las obras de fondo y algunas de surtido clasificadas por materias y orden alfabético de autores. Madrid: Librería Nacional y Extranjera de Fernando Fe.

Gallego, Josefa. 2005. "Estado actual de los estudios bibliográficos sobre el arte en España”. Scire 11 (1): 65-82.

Garzón, José A., Josep Alió y Miquel Artigas. 2012. NEBEA. Nuevo Ensayo de Bibliografía Española de Ajedrez. 1238-1938. Con apuntes para la bistoria del ajedrez español. Valencia: Romeditors.

Herrero, Cristina. 1996. "Metodología para un catálogo de libros del siglo XVI”. Revista General de Información y Documentación 6 (2): 11-50.

Hérubel, J. 1999. "Historical Bibliometrics: Its Purpose and Significance to the History of Disciplines". Libraries \& Culture 34 (4): 380-388.

Hocquart, L. 1810. Elémens Théoriques et practiques du jeu des echecs, avec des réflexions morales, politiques, historiques et militaires, relatives à ce jeu. París: chez Madame Veuve Hocquart. 
Índice Bibliográfico. 1933. Barcelona: Lib. Sintes.

Índice Bibliográfico de Obras de fondo y algunas de surtido. 1912. Madrid: Francisco Beltrán.

Lasker, Emanuel. 1920. Curso de Ajedrez. Buenos Aires: Librería de la Vda. de C. Bouret.

Llaverías, Amadeo. 1910. Catálogo de la Biblioteca del Gimnasio Colón. Barcelona: Antoni Palau y Dulcet.

Martínez, Ma. 1993-1995, dir. Diccionario bibliográfico de autores riojanos. Logroño: Gobierno de la Rioja.

Menéndez Pelayo, Marcelino. 1876. "De Re Bibliographica. Al señor D. Gumersindo Laverde Ruiz”. Revista Europea 8 (125): 65-73.

Olivera, Javier. 2011. "Prólogo", en Repertorio bibliográfico e inédito de la educación física y el deporte en España (1800-1939), Xavier Torrebadella, 9-13. Madrid: Fundación Universitaria Española.

Palau, Antoni. 1925. Llibreria Antiquaria de Antoni Palau. Catàleg núm. 27. Barcelona: Rafols.

Palau, Antoni. 1923-1945. Manual del librero bispano-americano: inventario bibliográfico... Barcelona: Palacete Palau Dulcet.

Paluzíe, José. 1943. Catálogo de la exposición bibliográfica de ajedrez. Barcelona: Casa Provincial de la Caridad.

Paluzíe, José. 1921. Manual de Ajedrez. Parte sexta (última). Miscelanea. Barcelona: s. e.

Paluzíe, José. 1912. Primer ensayo de bibliografía española de ajedrez. Barcelona: Hijos de Paluzíe.

Pastor, José Luis. 1995. La Educación Física en España: fuentes bibliográficas básicas. Alcalá de Henares: Universidad de Alcalá.

Pons, Francisco. 1898. Ensayo bio-bibliográfico sobre los Historiadores y Geógrafos arábgo-españoles. Madrid: Est. Tip. de San Francisco de Sales.

Preti, Jean-Louis. 1895. ABC des echecs. París: Numa Preti.

Punzano, Victoriano. 1981. "Pensamiento Bibliográfico de Menéndez Pelayo", en I Simposium Internacional de Literatura Hispanoamericana, 147-164. Santander: UIMP.

Ricart, Martín. 1897. Manual del ajdrecista. Barcelona: Saurí Sabater.

Sánchez, Juan Bautista. 1934. Diccionario Ilustrado de Ajedrez. Madrid: Imp. Cleto Vallinas.

Simón Díaz, José. 1994. Bibliografía de la literatura hispánica. Madrid: CSIC.

Simón Díaz, José. 1977. "Evolución y estado presente de la bibliografía española”. Primeras Jornadas de Bibliografía. Madrid: Fundación Universitaria Española, 15-31.

Simpson, Richard. 1863. Catalogue of Books on the origin, history, and practice of The Game of Chess. London: Reves.

Sobrequés, Santiago. 1966. Historia de España moderna y contemporánea. Barcelona: Vicens Vives.

Tolosa, José. 1892. Traité analytique du problème d'échecs. París: Numa Preti.

Torrebadella, Xavier. 2009. Contribución a la historia de la educación física en España. Estudio bio-bibliográfico en torno a la educación física y el deporte (1800-1939). Tesis doctoral no publicada, Universitat de Lleida.

Torrebadella, Xavier. 2014. "Rufino Blanco Sánchez y las fuentes bibliográficas de la educación física y el deporte en España”. RICYDE. Revista Internacional de Ciencias del Deporte 10 (37): 281-284. 
Torrebadella, Xavier y Antonio Nomdedeu. 2014. "Repertorio bibliográfico del fútbol en España (1900-1936). 121 obras para interpretar el impacto social del fútbol en la historia contemporánea”. Apunts. Educación física y deportes 1 (115): 7-32.

Torrebadella, Xavier. 2011. Repertorio bibliográfico inédito de la educación física y el deporte en España (1800-1939). Madrid: Fundación Universitaria Española.

Torrebadella, Xavier y Javier Olivera. 2012. "Las cien obras clave del repertorio bibliográfico español de la educación física y el deporte en su proceso de legitimación e institucionalización". Revista General de Información y Documentación (22): 119-168.

Torres, Isabel. 1992. "Quince años de trabajo bibliográfico: Bibliografía y Bibliografías en España (1977-1991)". Revista General de Información y Documentación: 139-159.

Vicents Vives, Jaime. 1942. Historia general moderna. Barcelona: Montaner y Simon.

Zarco del Valle, Bartolomé y José Sancho Rayón. 1863. "Prólogo", en Ensayo de una biblioteca española de libros raros y curiosos, 4 vols., José Gallardo, 1-3. Madrid: M. Rivadeneyra.

Para citar este texto:

Brasó i Rius, Jordi. 2018. "Los libros de ajedrez en España: (c. 17701899): bibliografía histórica”. Investigación Bibliotecológica: archivonomía, bibliotecología e información 32 (75): 47-67.

http://dx.doi.org/10.22201/iibi.24488321xe.2018.75.57943 